\title{
BLOW-UP PHENOMENA IN PARABOLIC PROBLEMS WITH TIME DEPENDENT COEFFICIENTS UNDER DIRICHLET BOUNDARY CONDITIONS
}

\author{
L. E. PAYNE AND G. A. PHILIPPIN
}

(Communicated by Michael Hitrik)

\begin{abstract}
A class of initial boundary value problems for the semilinear heat equation with time dependent coefficients is considered. Using a first order differential inequality technique, the influence of the data on the behaviour of the solutions (blow-up in finite or infinite time, global existence) is investigated. Lower and upper bounds are derived for the blow-up time when blow-up occurs.
\end{abstract}

\section{INTRODUCTION}

Blow-up phenomena of solutions to nonlinear parabolic equations have been assiduously investigated during the past decade. We refer the reader to the books of Straughan [20] and of Quittner and Souplet [19] as well as to the survey paper of Bandle and Brunner 2 for an account on this matter. Further contributions to the field are [1, 3]-18, 22]-24.

It is well known that the solutions may remain bounded for all time, or may blow up in finite or infinite time. When blow-up occurs at time $t^{\star}$, the evaluation of $t^{\star}$ is of great practical interest. Since $t^{\star}$ is usually not explicitly computable, we want to derive lower and upper bounds for $t^{\star}$.

The present paper investigates the blow-up phenomena of the solution $u(\mathbf{x}, t)$ of the following nonlinear parabolic problem:

$$
\left\{\begin{array}{l}
u_{t}=\Delta u+k(t) f(u), \quad \mathbf{x}=\left(x_{1}, \ldots, x_{N}\right) \in \Omega, \quad t \in\left(0, t^{\star}\right), \\
u(\mathbf{x}, t)=0, \quad \mathbf{x} \in \partial \Omega, \quad t \in\left(0, t^{\star}\right), \\
u(\mathbf{x}, 0)=u_{0}(\mathbf{x}), \quad \mathbf{x} \in \Omega,
\end{array}\right.
$$

where $\Omega$ is a bounded sufficiently smooth domain in $\mathbb{R}^{N}, N \geq 2$. The coefficient $k(t)$ is assumed nonnegative or strictly positive depending of the situation. The nonlinearity $f(u)$ is assumed to satisfy $f(0)=0$, and $f(u)>0$ for $u>0$. The initial data $u_{0}(\mathbf{x})$ is assumed nonnegative so that the solution $u(\mathbf{x}, t)$ of problem (1.1) is nonnegative by the maximum principle. More specific assumptions on $f$ and $k$ will be made later. The particular case of $k=$ const. has already been investigated by L. E. Payne and P. W. Schaefer in [13]. This note may therefore be regarded as a complement of their paper.

In Section 2, we derive conditions on the data of problem (1.1) sufficient to instigate the blow-up of $u(\mathbf{x}, t)$ and derive under these conditions an upper bound

Received by the editors July 9, 2011 and, in revised form, October 9, 2011.

2010 Mathematics Subject Classification. Primary 35K55, 35K61, 35B30, 35B44.

Key words and phrases. Parabolic problems, blow-up. 
for the blow-up time $t^{\star}$. In Section 3, we derive conditions on the data of problem (1.1) sufficient to insure the global existence of $u(\mathbf{x}, t)$. In Section 4 , we derive various lower bounds for $t^{\star}$, valid under appropriate assumptions on the data. In Sections 3 and 4, our argument makes use of the following Sobolev type inequality:

$$
\left(\int_{\Omega} v^{6} d \mathbf{x}\right)^{1 / 4} \leq \Gamma\left(\int_{\Omega}|\nabla v|^{2} d \mathbf{x}\right)^{3 / 4}, \quad \Gamma:=\frac{2 \cdot 3^{-3 / 4}}{\pi},
$$

valid in $\mathbb{R}^{3}$ for a nonnegative function $v$ that vanishes on $\partial \Omega$. We refer to [21 for a proof of (1.2). The results derived in Sections 3 and 4 are therefore restricted to the 3-dimensional space. Some extensions are derived in Section 5. The case of problem (1.1) under Neumann boundary conditions will be investigated in a forthcoming paper.

\section{Blow-UP OF $u(\mathbf{x}, t)$ IN Finite time $t^{*}$}

We first employ a method used by Kaplan 5] to obtain a condition which leads to blow-up at some finite time, and also leads to an upper bound for the blow-up time. Let $\lambda_{1}$ be the first eigenvalue and $\phi_{1}$ be the associated eigenfunction of the Dirichlet-Laplace operator defined as

$$
\begin{gathered}
\Delta \phi_{1}+\lambda_{1} \phi_{1}=0, \quad \phi_{1}>0, \quad \mathbf{x} \in \Omega ; \quad \phi_{1}=0, \quad \mathbf{x} \in \partial \Omega, \\
\int_{\Omega} \phi_{1} d \mathbf{x}=1 .
\end{gathered}
$$

Let the auxiliary function $\sigma(t)$ be defined in $\left(0, t^{\star}\right)$ as

$$
\sigma(t):=(k(t))^{1 /(p-1)} \int_{\Omega} u \phi_{1} d \mathbf{x}
$$

where $u(\mathbf{x}, t)$ is the solution of (1.1). We assume that

$$
f(0)=0, \quad f(s) \geq s^{p}, \quad s>0,
$$

for some $p>1$, and that $k(t)>0$ satisfies the condition

$$
\frac{k^{\prime}}{k} \geq \beta,
$$

for some constant $\beta$. With

$$
\gamma:=\lambda_{1}-\frac{\beta}{p-1}
$$

we compute

$$
\begin{aligned}
\sigma^{\prime}(t) & \geq \frac{\beta}{p-1} \sigma+k^{1 /(p-1)} \int_{\Omega} \phi_{1}\left[\left(\Delta u+k u^{p}\right] d \mathbf{x}\right. \\
& =-\gamma \sigma+k^{p /(p-1)} \int_{\Omega} \phi_{1} u^{p} d \mathbf{x} .
\end{aligned}
$$

Moreover, making use of Hölder's inequality, we have in view of (2.2),

$$
\int_{\Omega} \phi_{1} u d \mathbf{x} \leq\left(\int_{\Omega} \phi_{1} u^{p} d \mathbf{x}\right)^{1 / p} .
$$

Combining (2.8) and (2.7), we obtain the first order differential inequality

$$
\sigma^{\prime}(t) \geq-\gamma \sigma(t)+(\sigma(t))^{p}, \quad t \in\left(0, t^{\star}\right) .
$$


Integrating (2.9), we obtain

$$
(\sigma(t))^{1-p} \leq \eta(t):=\left\{\begin{array}{l}
\frac{1}{\gamma}+\delta e^{(p-1) \gamma t}, \quad \gamma \neq 0, \\
(\sigma(0))^{1-p}-(p-1) t, \quad \gamma=0
\end{array}\right.
$$

with

$$
\delta:=(\sigma(0))^{1-p}-\frac{1}{\gamma}, \quad \gamma \neq 0
$$

If $\eta\left(T_{1}\right)=0$ for some $T_{1}>0$, then $\sigma(t)$ blows up at time $t^{\star}<T_{1}$. This leads to the following result.

Theorem 1. Let $u(\mathbf{x}, t)$ be the solution of problem (1.1). Then the auxiliary function $\sigma(t)$ defined in (2.3) blows up at time $t^{\star}<T_{1}$ with

$$
T_{1}:=\left\{\begin{array}{l}
\frac{1}{\gamma(p-1)} \log \left(-\frac{1}{\gamma \delta}\right) \quad \text { if } 0<\gamma(\sigma(0))^{1-p}<1 \\
\frac{1}{(p-1)(\sigma(0))^{p-1}} \quad \text { if } \quad \gamma \leq 0 .
\end{array}\right.
$$

Another upper bound for the blow-up time could be obtained by a variation of the concavity method of Levine [7. For $k(t)=1$, sufficient conditions leading to the blow-up of $u(\mathbf{x}, t)$ have been etablished by Philippin and Proytcheva in [17. Their approach may be adapted when $k(t)$ is not constant. This leads to the following result.

Theorem 2. Let $u(\mathbf{x}, t)$ be the solution of problem (1.1). Let $\psi(t)$ and $\zeta(t)$ be auxiliary functions defined as follows:

$$
\begin{gathered}
\psi(t):=\int_{\Omega} u^{2}(\mathbf{x}, t) d \mathbf{x}, \\
\zeta(t):=\int_{\Omega}\left\{k(t) F(u)-\frac{1}{2}|\nabla u|^{2}\right\} d \mathbf{x},
\end{gathered}
$$

with

$$
F(u):=\int_{0}^{u} f(s) d s .
$$

Assume the following conditions on $f$ and $k$ :

$$
\begin{gathered}
k(0)>0, \quad k^{\prime}(t) \geq 0, \quad t>0, \\
s f(s) \geq \frac{1}{2}(4+\alpha) F(s),
\end{gathered}
$$

where $\alpha$ is a positive parameter. Moreover we assume that the initial data satisfy the condition

$$
\zeta(0)=\int_{\Omega}\left\{k(0) F\left(u_{0}\right)-\frac{1}{2}\left|\nabla u_{0}\right|^{2}\right\} d \mathbf{x}>0 .
$$

Then we conclude that $u(\mathbf{x}, t)$ blows up at some finite time $t^{\star}<T_{2}$ with

$$
T_{2}:=\frac{4}{\alpha(\alpha+4)} \psi(0)(\zeta(0))^{-1} .
$$

We refer to [17] for the proof of Theorem 2. 
3. Conditions for global existence of $u(\mathbf{x}, t)$

In this section, we assume that the data of problem (1.1) satisfy the following two conditions:

$$
\begin{gathered}
0 \leq f(s) \leq s^{p}, \quad s>0, \quad p=\text { const. }>1 \\
k(t)>0, \quad \frac{k^{\prime}(t)}{k(t)} \leq \beta, \quad t \geq 0
\end{gathered}
$$

for some positive constant $\beta$ and consider the auxiliary function $\Phi(t)$ defined as

$$
\Phi(t):=M^{-1}(k(t))^{2 n} \int_{\Omega} u^{2 n(p-1)} d \mathbf{x}, \quad t \in\left(0, t^{\star}\right),
$$

with

$$
M:=(k(0))^{2 n} \int_{\Omega} u_{0}^{2 n(p-1)} d \mathbf{x} .
$$

In (3.3), (3.4), $n$ is a parameter subject to the restrictions

$$
n(p-1) \geq 1, \quad n>3 / 4 .
$$

For instance we may choose $n=1 /(p-1)$ if $p \in(1,2)$, or $n=1$ if $p \geq 2$. For convenience we set

$$
v(\mathbf{x}, t):=u^{n(p-1)}
$$

and compute

$$
\Phi^{\prime}(t)=2 n \frac{k^{\prime}}{k} \Phi+2 n(p-1) M^{-1} k^{2 n} \int_{\Omega} u^{2 n(p-1)-1}[\Delta u+k(t) f(u)] d \mathbf{x} .
$$

With

$$
\int_{\Omega} u^{2 n(p-1)-1} \Delta u d \mathbf{x}=-\frac{2 n(p-1)-1}{n^{2}(p-1)^{2}} \int_{\Omega}|\nabla v|^{2} d \mathbf{x},
$$

we obtain, thanks to $(3.1),(3.2)$,

$$
\begin{aligned}
\Phi^{\prime}(t) \leq 2 n \beta \Phi & +2 n(p-1) k^{2 n+1} M^{-1} \int_{\Omega} v^{2+\frac{1}{n}} d \mathbf{x} \\
& -\frac{2[2 n(p-1)-1]}{n(p-1)} k^{2 n} M^{-1} \int_{\Omega}|\nabla v|^{2} d \mathbf{x} .
\end{aligned}
$$

Making use of Hölder's inequality

$$
\int_{\Omega} v^{2+\frac{1}{n}} d \mathbf{x} \leq\left(\int_{\Omega} v^{2} d \mathbf{x}\right)^{(4 n-1) / 4 n}\left(\int_{\Omega} v^{6} d \mathbf{x}\right)^{1 / 4 n}
$$

followed by the Sobolev type inequality (1.2), we obtain

$$
\begin{aligned}
k^{2 n+1} \int_{\Omega} v^{2+\frac{1}{n}} d \mathbf{x} & \leq k^{2 n+1}\left(\int_{\Omega} v^{2} d \mathbf{x}\right)^{(4 n-1) / 4 n}\left(\int_{\Omega}|\nabla v|^{2} d \mathbf{x}\right)^{3 / 4 n} \Gamma^{1 / n} \\
& =\Gamma^{1 / n} M^{(4 n-1) / 4 n} \Phi^{(4 n-1) / 4 n}\left(k^{2 n} \int_{\Omega}|\nabla v|^{2} d \mathbf{x}\right)^{3 / 4 n},
\end{aligned}
$$


where $\Gamma$ is defined in (1.2). Combining (3.11) and (3.9), we obtain

$\Phi^{\prime}(t)$

$$
\begin{aligned}
& \leq 2 n \beta \Phi-\frac{2[2 n(p-1)-1]}{n(p-1)} M^{-1} k^{2 n} \int_{\Omega}|\nabla v|^{2} d \mathbf{x} \\
& +2 n(p-1) \Gamma^{1 / n} M^{1 / 2 n} \Phi^{(4 n-1) / 4 n}\left(M^{-1} k^{2 n} \int_{\Omega}|\nabla v|^{2} d \mathbf{x}\right)^{3 / 4 n} \\
& =2 n \beta \Phi+2 n\left(\lambda^{-1} k^{2 n} M^{-1} \int_{\Omega}|\nabla v|^{2} d \mathbf{x}\right)^{3 / 4 n} \\
& \times\left\{\lambda^{3 / 4 n}\left(p-1 \Gamma^{1 / n} \Phi^{\frac{(4 n-1)}{4 n}} M^{1 / 2 n}-\frac{2 n(p-1)-1}{n^{2}(p-1)} \lambda\left(M^{-1} k^{2 n} \lambda^{-1} \int_{\Omega}|\nabla v|^{2} d \mathbf{x}\right)^{\frac{(4 n-3)}{4 n}}\right\}\right.
\end{aligned}
$$

with arbitrary $\lambda \neq 0$. Choosing $\lambda:=\lambda_{1}$, the first eigenvalue of problem (2.1), we have

$$
\int_{\Omega}|\nabla v|^{2} d \mathbf{x} \geq \lambda_{1} \int_{\Omega} v^{2} d \mathbf{x}
$$

by the Rayleigh principle. Making use of (3.13) in the last factor of (3.12), we obtain the inequality

$$
\begin{aligned}
\Phi^{\prime} & \leq 2 n \beta \Phi+2 n\left(\lambda_{1}^{-1} k^{2 n} M^{-1} \int_{\Omega}|\nabla v|^{2} d \mathbf{x}\right)^{3 / 4 n} \Phi^{(4 n-3) / 4 n} \\
& \times\left\{\nu \Phi^{1 / 2 n}-(\mu+\beta)\right\},
\end{aligned}
$$

with

$$
\nu:=(p-1) \lambda_{1}^{3 / 4 n} \Gamma^{1 / n} M^{1 / 2 n}, \quad \mu:=\frac{2 n(p-1)-1}{n^{2}(p-1)} \lambda_{1}-\beta .
$$

Suppose that $\beta$ is small enough to satisfy the condition

$$
\mu>0 \text {, }
$$

and that the initial data are small enough to satify the condition

$$
\nu-\mu<0 \text {. }
$$

Then either $\nu(\Phi(t))^{1 / 2 n}-\mu$ remains negative for all time, or there exists a first time $t_{0}$ such that

$$
\nu\left(\Phi\left(t_{0}\right)\right)^{1 / 2 n}-\mu=0 .
$$

For $t \in\left(0, t_{0}\right)$, the last factor in (3.14) is then negative, and we may use again (3.13) in (3.14) to obtain the differential inequality

$$
\Phi^{\prime}(t) \leq 2 n \Phi\left\{\nu \Phi^{1 / 2 n}-\mu\right\} \leq 0, \quad t \in\left(0, t_{0}\right) .
$$

It follows from (3.19) that $\Phi(t)$ is nonincreasing on $\left(0, t_{0}\right)$, so that (3.18) cannot hold. We then conclude that (3.19) is valid for all time $t>0$. Integrating this differential inequality, we obtain

$$
\Phi(t) \leq\left\{\left(1-\frac{\nu}{\mu}\right) e^{\mu t}+\frac{\nu}{\mu}\right\}^{-2 n}, \quad t>0 .
$$

This result is summarized in the next theorem. 
Theorem 3. Let $\Omega$ be a bounded domain in $\mathbb{R}^{3}$, and assume that the data of problem (1.1) satisfy the conditions (3.1), (3.2), (3.16), (3.17). Then the auxiliary function $\Phi(t)$ defined in (3.3) satisfies (3.20), and $u(\mathbf{x}, t)$ exists for all time $t>0$.

\section{LOWER BOUNDS FOR $t^{\star}$}

In this section some lower bounds for $t^{\star}$ will be derived for the possible blow-up time of the solution $u(\mathbf{x}, t)$ of problem (1.1). Going back to the first inequality (3.12) and making use of the inequality

$$
\begin{aligned}
\Phi^{(4 n-1) / 4 n} J^{3 / 4 n} & =\left(c^{3 /(4 n-3)} \Phi^{(4 n-1) /(4 n-3)}\right)^{(4 n-3) / 4 n}\left(c^{-1} J\right)^{3 / 4 n} \\
& \leq \frac{4 n-3}{4 n} c^{3 /(4 n-3)} \Phi^{(4 n-1) /(4 n-3)}+\frac{3}{4 n c} J,
\end{aligned}
$$

with $J:=M^{-1}(k(t))^{2 n} \int_{\Omega}|\nabla v|^{2} d \mathbf{x}$, valid for arbitrary $c>0$, we obtain

$$
\begin{aligned}
\Phi^{\prime} & \leq 2 n \beta \Phi+\frac{1}{2}(4 n-3)(p-1) M^{1 / 2 n} \Gamma^{1 / n} c^{3 /(4 n-3)} \Phi^{(4 n-1) /(4 n-3)} \\
& +\left\{\frac{3(p-1)}{2 c} M^{1 / 2 n} \Gamma^{1 / n}-\frac{2[2 n(p-1)-1]}{n(p-1)}\right\} J .
\end{aligned}
$$

Selecting

$$
c:=\frac{3 n(p-1)^{2}}{4[2 n(p-1)-1]} M^{1 / 2 n} \Gamma^{1 / n}
$$

the last term in (4.2) vanishes and we obtain the differential inequality

$$
\Phi^{\prime}(t) \leq 2 n \beta \Phi+c_{1} \Phi^{(4 n-1) /(4 n-3)},
$$

with

$$
\begin{gathered}
c_{1}:=c_{0} M^{2 /(4 n-3)}, \\
c_{0}:=\frac{1}{2}(4 n-3)(p-1)\left(\frac{3 n(p-1)^{2}}{4[2 n(p-1)-1]}\right)^{3 /(4 n-3)} \Gamma^{4 /(4 n-3) .}
\end{gathered}
$$

Integrating this differential inequality, we obtain

$$
(\Phi(t))^{-2 /(4 n-3)} \geq\left(1+\frac{c_{1}}{2 n \beta}\right) \exp \left(-\frac{4 n \beta}{4 n-3} t\right)-\frac{c_{1}}{2 n \beta} .
$$

We then conclude that if $\Phi(t)$ blows up at some time $t^{\star}$, we have

$$
t^{\star} \geq t_{1}:=\frac{4 n-3}{4 n \beta} \log \left(1+\frac{2 n \beta}{c_{1}}\right) .
$$

We note again that an appropriate choice of $n$ is $n=1$ for $p \geq 2$, or $n=(p-1)^{-1}$ for $p \in(1,2)$.

To establish a lower bound for $t^{\star}$ that does not require the condition (3.2), we define the auxiliary function

$$
\Theta(t):=M_{0}^{-1} \int_{\Omega} u^{2 n(p-1)} d \mathbf{x}
$$

with

$$
M_{0}:=\int_{\Omega} u_{0}^{2 n(p-1)} d \mathbf{x}
$$


Of course we keep the restrictions (3.5) on the parameter $n$. Assuming (3.1), we compute

$$
\Theta^{\prime}(t) \leq 2 n(p-1) M_{0}^{-1} k \int_{\Omega} v^{2+\frac{1}{n}} d \mathbf{x}-\frac{2[2 n(p-1)-1]}{n(p-1)} M_{0}^{-1} \int_{\Omega}|\nabla v|^{2} d \mathbf{x},
$$

where $v(\mathbf{x}, t)$ is defined in (3.6). Proceeding as before leads to the differential inequality

$$
\Theta^{\prime}(t) \leq c_{2}(k(t))^{4 n /(4 n-3)} \Theta^{(4 n-1) /(4 n-3)},
$$

with

$$
c_{2}:=c_{0} M_{0}^{2 /(4 n-3)},
$$

where $c_{0}$ is defined in (4.6). Integrating (4.12), we obtain

$$
(\Theta(t))^{-2 /(4 n-3)} \geq 1-\frac{2 c_{2}}{4 n-3} \int_{0}^{t}(k(\tau))^{4 n /(4 n-3)} d \tau .
$$

We then conclude that $\Theta(t)$ remains bounded for all time if the condition

$$
\int_{0}^{\infty}(k(t))^{4 n /(4 n-3)} d t<\frac{4 n-3}{2 c_{2}}
$$

is satisfied. On the other hand, if $\Theta(t)$ blows up at some finite time $t^{\star}$, then $t^{\star}>t_{2}$, with

$$
\int_{0}^{t_{2}}(k(t))^{4 n /(4 n-3)} d t=\frac{4 n-3}{2 c_{2}} .
$$

These results are summarized in the next theorem.

Theorem 4. Let $u(\mathbf{x}, t)$ be the solution of problem (1.1) in a bounded domain $\Omega \subset \mathbb{R}^{3}$.

a) Assume that $f$ satisfies (3.1), (3.2). Then the auxiliary function $\Phi(t)$ defined in (3.3) is bounded above by (4.7), and blows up at time $t^{\star}>t_{1}$ defined in (4.8) when blow-up occurs.

b) Assume that $f$ satisfies (3.1). We then conclude that the auxiliary function $\Theta(t)$ defined in (4.9) remains bounded if the condition (4.15) is satisfied; otherwise $\Theta(t)$ blows up at time $t^{\star}>t_{2}$ defined in (4.16) when blow-up occurs.

\section{Some EXtensions}

In this section we consider the more general problem

$$
\left\{\begin{array}{l}
\frac{1}{k_{1}(t)} u_{t}=k_{2}(t) \Delta u+k_{3}(t) f(u), \quad \mathbf{x} \in \Omega, \quad t \in\left(0, t^{\star}\right), \\
u(\mathbf{x}, t)=0, \quad \mathbf{x} \in \partial \Omega, \quad t \in\left(0, t^{\star}\right), \\
u(\mathbf{x}, 0)=u_{0}(\mathbf{x}) \geq 0, \quad \mathbf{x} \in \Omega,
\end{array}\right.
$$

where the given differentiable functions $k_{1}, k_{2}, k_{3}$ are assumed positive. We consider the auxiliary function

$$
\chi(t):=Q^{-1} K(t) \int_{\Omega} u^{2 n(p-1)} d \mathbf{x}
$$


with

$$
\begin{gathered}
K(t):=\left(k_{1}(t)\right)^{(4 n-3) / 2}\left(k_{2}(t)\right)^{-3 / 2}\left(k_{3}(t)\right)^{2 n}, \\
Q:=K(0) \int_{\Omega} u_{0}^{2 n(p-1)} d \mathbf{x},
\end{gathered}
$$

where $n$ is a parameter subject to the restrictions (3.5). Assuming (3.1) and

$$
\left(\frac{1}{2 n}\right) \frac{K^{\prime}(t)}{K(t)}=\left(\frac{4 n-3}{4 n}\right) \frac{k_{1}^{\prime}}{k_{1}}-\left(\frac{3}{4 n}\right) \frac{k_{2}^{\prime}}{k_{2}}+\frac{k_{3}^{\prime}}{k_{3}} \leq \beta
$$

for some $\beta \in \mathbb{R}$, we have the following differential inequality:

$$
\chi^{\prime}(t) \leq 2 n \beta \chi+c_{3} \chi^{(4 n-1) /(4 n-3)},
$$

valid for $\Omega \in \mathbb{R}^{3}$, with

$$
c_{3}:=c_{0} Q^{2 /(4 n-3)}
$$

Integrating (5.6), we obtain

$$
(\chi(t))^{-2 /(4 n-3)} \geq \epsilon(t):=\left\{\begin{array}{l}
1-\frac{2 c_{3}}{4 n-3} t, \quad \beta=0, \\
\left(1+\frac{c_{3}}{2 n \beta}\right) \exp \left(-\frac{4 n \beta}{4 n-3} t\right)-\frac{c_{3}}{2 n \beta}, \quad \beta \neq 0 .
\end{array}\right.
$$

We then conclude that if $\chi(t)$ blows up at some $t^{\star}$, we have

$$
t^{\star} \geq t_{3}:=\left\{\begin{array}{l}
\frac{4 n-3}{2 c_{3}}, \quad \beta=0, \\
\frac{4 n-3}{4 n \beta} \log \left(1+\frac{2 n \beta}{c_{3}}\right), \quad \beta \neq 0 .
\end{array}\right.
$$

For $\beta \neq 0$, the value of $t_{3}$ in (5.9) makes sense only in the following two cases: (i) $\beta>0$, (ii) $\beta<0$ and $1+2 n \beta / c_{3}>0$. In all other cases the solution $u(\mathbf{x}, t)$ of (5.1) exists for all time $t>0$.

To establish a lower bound for $t^{\star}$ that does not require the condition (5.5), we consider again the auxiliary function $\Theta(t)$ defined in (4.9), (4.10). Assuming (3.1), we obtain the differential inequality

$$
\begin{aligned}
\Theta^{\prime}(t) & \leq c_{2} k_{1}(t)\left(k_{2}(t)\right)^{-3 /(4 n-3)}\left(k_{3}(t)\right)^{4 n /(4 n-3)} \Theta^{(4 n-1) /(4 n-3)} \\
& =c_{2}(K(t))^{2 /(4 n-3)} \Theta^{(4 n-1) /(4 n-3)},
\end{aligned}
$$

with $K(t)$ defined in (5.3) and $c_{2}$ defined in (4.13). It then follows that

$$
(\Theta(t))^{-2 /(4 n-3)} \geq 1-\frac{2 c_{2}}{4 n-3} \int_{0}^{t}(K(\tau))^{2 /(4 n-3)} d \tau
$$

We then conclude that $\Theta(t)$ remains bounded for all time if the condition

$$
\int_{0}^{\infty}(K(t))^{2 /(4 n-3)} d t<\frac{4 n-3}{2 c_{2}}
$$

is satisfied. On the other hand, if $\Theta(t)$ blows up at some finite time $t^{\star}$, then $t^{\star}>t_{4}$ with

$$
\int_{0}^{t_{4}}(K(t))^{2 /(4 n-3)} d t=\frac{4 n-3}{2 c_{2}} .
$$

These results are summarized in the next theorem. 
Theorem 5. Let $u(\mathbf{x}, t)$ be the solution of problem (5.1) in a bounded domain $\Omega \subset \mathbb{R}^{3}$

a) Assume (3.1), (5.5), (3.5). Then the auxiliary function $\chi(t)$ defined in (5.2) blows up at time $t^{\star}$ bounded below by $t_{3}$ defined in (5.9) in the two cases (i) and (ii). Otherwise $u(\mathbf{x}, t)$ exists for all time $t>0$.

b) Assume (3.1). We then conclude that the auxiliary function $\Theta(t)$ defined in (4.9), (4.10) remains bounded if condition (5.12) is satisfied. Otherwise $\Theta(t)$ blows up at time $t^{\star}>t_{4}$ defined in (5.13) when blow-up occurs.

To conclude this paper we show how problem (5.1) may be reduced to problem (1.1). To this end we replace the time variable by the new variable

$$
z(t):=\int_{0}^{t} k_{1}(\tau) k_{2}(\tau) d \tau
$$

The differential equation in (5.1) then takes the following form:

$$
u_{z}(\mathbf{x}, z)=\Delta u+\kappa(z) f(u), \quad \mathbf{x} \in \Omega, \quad z \in\left(0, z^{\star}\right),
$$

with $z^{\star}=z\left(t^{\star}\right)$ and with

$$
\kappa(z):=\frac{k_{3}(t(z))}{k_{2}(t(z))} .
$$

The results derived in Sections 2, 3, and 4 are therefore applicable to the more general problem (5.1).

\section{REFERENCES}

[1] Ball, J. M., Remarks on blow-up and nonexistence theorems for nonlinear evolution equations. Quart. J. Math. Oxford, 28, 473-486, (1977). MR0473484 (57:13150)

[2] Bandle, C., Brunner, H., Blowup in diffusion equations: a survey, J. Comput. Appl. Math., 97, 3-22, (1998). MR.1651764 (99g:35061)

[3] Enache, C., Blow-up phenomena for a class of quasilinear parabolic problems under Robin boundary conditions. Appl. Math. Letters, 24, 288-292, (2011). MR2741031(2011h:35145)

[4] Enache, C., Lower bounds for blow-up time in some non-linear parabolic problems under Neumann boundary conditions. Glasgow Math. J., 53, 569-575, (2011). MR2822799(2012i:35174)

[5] Kaplan, S., On the growth of solutions of quasilinear parabolic equations. Comm. Pure Appl. Math., 16, 305-330, (1963). MR0160044 (28:3258)

[6] Kielhöfer, H., Halbgruppen und semilineare Anfangs-randwert-probleme. Manuscripta Math., 12, 121-152, (1974). MR0344681 (49:9420)

[7] Levine, H. A., Some nonexistence and instability theorems for solutions of formally parabolic equations of the form $P u_{t}=-A u+F(u)$, Arch. Rat. Mech. Anal., 51, 371-386, (1973). MR0348216(50:714)

[8] Payne, L. E., Philippin, G. A., Schaefer, P. W., Bounds for blow-up time in nonlinear parabolic problems. J. Math. Anal. Appl., 338, 438-447, (2008). MR2386428(2009c:35233)

[9] Payne, L. E., Philippin, G. A., Schaefer, P. W., Blow-up phenomena for some nonlinear parabolic problems. Nonlinear Anal., 69, 3495-3502, (2008). MR2450554 (2010a:35105)

[10] Payne, L. E., Philippin, G. A., Vernier-Piro, S., Blow-up phenonena for a semilinear heat equation with nonlinear boundary condition, I. Z. Angew. Math. Phys., 61, 999-1007, (2010). MR2738300

[11] Payne, L. E., Philippin, G. A., Vernier-Piro, S., Blow-up phenonena for a semilinear heat equation with nonlinear boundary condition, II. Nonlinear Anal., 73, 971-978, (2010). MR.2653764(2011d:35264)

[12] Payne, L. E., Schaefer, P. W., Lower bound for blow-up time in parabolic problems under Neumann conditions. Appl. Anal., 85, 1301-1311, (2006). MR2263927 (2007f:35147)

[13] Payne, L. E., Schaefer, P. W., Lower bound for blow-up time in parabolic problems under Dirichlet conditions. J. Math. Anal. Appl., 328, 1196-1205, (2007). MR2290045 (2007k:35239) 
[14] Payne, L. E., Schaefer, P. W., Blow-up phenomena for some nonlinear parabolic systems. Int. J. Pure Appl. Math., 48, 193-202, (2008). MR2463922(2009k:35114)

[15] Payne, L. E., Schaefer, P. W., Bounds for the blow-up time for the heat equation under nonlinear boundary conditions. Proc. Roy. Soc. Edinburgh, 139 A, 1289-1296, (2009). MR2557324 (2010k:35268)

[16] Payne, L. E., Song, J. C., Lower bounds for blow-up time in a nonlinear parabolic problem, J. Math. Anal. Appl., 354, 394-396, (2009). MR2510449(2010b:35226)

[17] Philippin, G. A., Proytcheva, V., Some remarks on the asymptotic behaviour of the solutions of a class of parabolic problems. Math. Meth. Appl. Sci., 29, 297-307, (2006). MR2191431 (2007g:35109)

[18] Quittner, P., On global existence and stationary solutions of two classes of semilinear parabolic equations. Comm. Math. Univ. Carolin., 34, 105-124, (1993). MR1240209 (94g:35127)

[19] Quittner, P., Souplet, P., Superlinear parabolic problems. Blow-up, global existence and steady states. Birkhäuser, Basel, (2007). MR2346798 (2008f:35001)

[20] Straughan, B., Explosive Instabilities in Mechanics. Springer, Berlin, (1998). MR.1637904 (99k:35175)

[21] Talenti, G., Best constant in Sobolev inequality. Annali Mat. Pura Appl., 110, 353-372, (1976). MR0463908 (57:3846)

[22] Vazquez, J. L., The problems of blow-up for nonlinear heat equations. Complete blow-up and avalanche formation. Atti Accad. Naz. Lincei Cl. Sci. Fis. Mat. Natur. Rend. Lincei (9) Mat. Appl., 15, 281-300, (2004). MR2148886(2006b:35172)

[23] Weissler, F. B., Local existence and nonexistence for semilinear parabolic equations in $L^{p}$. Indiana Univ. Math. J., 29, 79-102, (1980). MR554819(81c:35072)

[24] Weissler, F. B., Existence and nonexistence of global solutions for a heat equation, Israël $J$. Math., 38(1-2), 29-40, (1981). MR.599472 (82g:35059)

Department of Mathematics, Cornell University, IthacA, New York 14853

Département de Mathématiques et de Statistique, Université Laval, Québec, Canada G1V 0A6

E-mail address: gphilip@mat.ulaval.ca 